\title{
$17 \beta$-estradiol and xenoestrogens reveal synergistic effect on mitochondria of human sperm
}

\author{
Izabela Skibińska' , Magdalena Jendraszak' ${ }^{1}$, Karolina Borysiak , Piotr Jędrzejczak ${ }^{2}$, \\ Małgorzata Kotwicka ${ }^{1}$ \\ ${ }^{1}$ Department of Cell Biology, Poznan University of Medical Sciences, Poznan, Poland \\ ${ }^{2}$ Division of Infertility and Reproductive Endocrinology, Department of Gynecology, Obstetrics and Gynecological Oncology, \\ Poznan University of Medical Sciences, Poznan, Poland
}

\begin{abstract}
Objectives: The aim of the study was to investigate the influence of $17 \beta$-estradiol (main endogenous estrogen) and selected xenoestrogens (genistein, bisphenol-A), individually and in combination, on the mitochondrial function of human spermatozoa. In natural environment, human beings are exposed to multiple xenoestrogens, so their impact is combined with endogenous steroids.

Material and methods: The effects of ligands on human spermatozoa were assessed regarding the following phenomena: spermatozoa vitality (propidium iodide staining), phosphatidylserine membrane translocation (staining with annexin $\mathrm{V}$ marked with fluorescein), mitochondrial membrane potential (using JC-1 fluorochrome), and production of superoxide anion in mitochondria (using MitoSOX RED dye).

Results: Two-hour incubation of spermatozoa with $17 \beta$-estradiol genistein, and bisphenol-A neither altered cell vitality nor stimulated phosphatidylserine membrane translocation. Incubation of spermatozoa with $17 \beta$-estradiolor bisphenol-A separately, as well as incubation with the three ligands simultaneously, resulted in altered mitochondrial membrane potential. Spermatozoa incubation with the three ligands significantly increased the mitochondrial superoxide anion level.

Conclusions: It seems safe to conclude that human spermatozoa mitochondria are target cell structures for both, $17 \beta$-estradiol and xenoestrogens. The reaction to the $17 \beta$-estradiol and xenoestrogens mixture suggests a synergistic mechanism of action. Xenoestrogens may increase the sensitivity of spermatozoa to $17 \beta$-estradiol.
\end{abstract}

Key words: human spermatozoa, mitochondria, 17 $\beta$-estradiol, genistein, bisphenol-A

Ginekologia Polska 2016; 87, 5: 360-366

\section{INTRODUCTION}

Infertility is a worldwide reproductive health problem, affecting about $15-20 \%$ of the reproductive-age population. According to various authors, the male factor may be the cause of $30-60 \%$ of infertility cases. Approximately $60-75 \%$ of infertile males do not receive any clear diagnosis so their infertility is referred to as idiopathic or unexplained. However, a steadily increasing number of reports have indicated that male exposure to environmental contaminants may impair human reproduction [1-3]. Some of these chemicals (natural or synthetic) can mimic natural hormones and are capable of modulating or disrupting the endocrine system. Endocrine disrupting chemicals can be grouped into three types: (1) synthetic hormones (e.g. 17a-ethinylestradiol), (2) natural compounds (e.g. genistein), and (3) man-made chemicals (e.g. bisphenol-A, BPA).

Xenoestrogens probably exert their biological effects through involvement in the signal transduction pathways used by endogenous estrogens. Estrogens affect the target cells through the genomic and nongenomic mechanism via ESR1 and ESR2 estrogen receptors. In the classical genomic mechanism, ESR function as transcription factors. Fast effects observed within a number of cells, a few seconds after stimulation with estrogens, indicate a nongenomic mechanism of their action $[4,5]$. 
Experiments on somatic cells indicated that mitochondria are target organelles for estrogen [6,7]. It is postulated that mitochondria constitute a specific estrogen reservoir [8]. Moreover, expression of both, ESR1 and ESR2 was shown in the mitochondria of somatic cells [9]. It is suggested that ESR can serve as a transcription factor for mitochondrial DNA genes [10]. It has also been found that estrogens can stimulate the expression of genes encoding mitochondrial respiratory chain proteins $[11,12]$. Estrogens have been suggested to inhibit sodium-dependent efflux of calcium ions from mitochondria. As a consequence of the increase in mitochondrial calcium ion concentration, enhanced synthesis of reactive oxygen species (ROS) such as superoxide anion $\left(\mathrm{O}_{2}{ }^{*-}\right)$ occurs $[13,14]$. Moreover, also estrogen-dependent tissues seem to be more susceptible to oxidative stress, resulting in DNA damage and, consequently, in higher mutation rates [15].

It was postulated that spermatozoa are also target cells for estrogens. It has been indicated that both forms of $\mathrm{ESR}_{\mathrm{s}}$ are present in spermatozoa but reports concerning their localization remain ambiguous [16-19]. We observed that ESR1 and ESR2 expression in human spermatozoa was strong in the midpiece region [in press], which can lead to the assumption that estrogen receptors are present also in spermatozoal mitochondria, similarly to their proven presence in somatic cells. Results obtained by Solakidi et al., with the use of the CMX mitochondrial marker, seem to confirm this hypothesis [18]. It cannot be excluded that xenoestrogens can also affect the functions of these organelles.

BPA binds to both, ESR1 and ESR2, with approximately 10-fold higher affinity to ESR2. BPA is observed to be a very weak environmental estrogen because of its low ESR affinity (affinity of BPA for $\mathrm{ESR}_{\mathrm{s}}$ is 10,000-100,000-fold weaker than of E2). However, it was detected that BPA can stimulate cellular responses at very low concentrations (reviewed [20]).

Genistein, classified as isoflavone, can also influence gene transcription which depends on ESR activation. Various studies have shown that estrogenic potency of genistein for ESR2 is much higher (by 30-fold) than for ESR1. Despite that fact, genistein induces ESR1-mediated results more effectively [21,22]. Binding affinity of genistein and other isoflavones is more significant than that of synthetic xenoestrogens, such as the abovementioned BPA [23].

In light of these observations, we aimed to investigate the influence of bisphenol-A and genistein on the mitochondrial function of human spermatozoa, based on the analysis of changes in mitochondrial membrane potential and detection of mitochondrial superoxide anion. Due to the fact that in natural environment human beings are not exposed to a single chemical compound, but rather to a complex of them, mixtures of E2, genistein, and BPA were examined.

\section{MATERIAL AND METHODS \\ Preparation of human spermatozoa}

Semen samples obtained from 15 normozoospermic men were analyzed according to the 2010 WHO criteria [24]. A 3-5-day period of sexual abstinence was required prior to obtaining the material. High-motility sperm cells were isolated with the use of the swim-up technique [25]. Ham's F-10 medium served as sperm cell extender.

Changes in different parameters of spermatozoa such as sperm vitality, phosphatidylserine membrane translocation, mitochondrial membrane potential, and mitochondrial superoxide anion level were then analyzed. The two-step model of analysis was applied in this study: (1) incubation of sperm cells with single entities at the following final concentrations: $10^{-10}$, $10^{-8}$ or $10^{-6} \mathrm{~mol} / \mathrm{L}$, and (2) cell exposure to mixtures of endogenous estrogen and xenoestrogens (E2, genistein and BPA), each of those used at a concentration of $10^{-10} \mathrm{~mol} / \mathrm{L}$. Sperm cells stimulated by Ham's F-10 medium were used as controls.

\section{Sperm vitality and phosphatidylserine membrane translocation}

Annexin-V labeled with fluorescein (AnV-FLUOS) (Molecular Diagnostics, Darmstadt, Germany) was used to determine membrane translocation of phosphatidylserine (PST) from the inner to the outer layer of plasma membrane. Viable and dead spermatozoa were distinguished by simultaneous staining with the use of propidium iodide (PI) at a final concentration of $0.125 \mu \mathrm{g} / \mathrm{L}$ (Sigma-Aldrich, St. Louis, MO). Double staining was conducted according to the manufacturer's recommendations.

\section{Detection of mitochondrial membrane potential}

5,5'6,6'-tetrachloro-1,1'3,3'-tetraethylbenzimidazolocarbocyanine iodide (JC-1; Molecular Probes, USA) was used to evaluate mitochondrial membrane potential $\left(\Delta \Psi_{m}\right)$ of spermatozoa. Depending on either low $\left(\Delta \Psi_{\mathrm{m}}<80-100 \mathrm{mV}\right)$ or high $\left(\Delta \Psi_{\mathrm{m}}>80-100 \mathrm{mV}\right)$ mitochondrial potential of the cells, JC-1 forms monomers or aggregates and emits either green (wavelength of 525 to $530 \mathrm{~nm}$ ) or red-orange (wavelength of $590 \mathrm{~nm}$ ) fluorescence, respectively. The excitation of fluorescence in both cases takes place at $488 \mathrm{~nm}$. Prior to cell staining, the dilution of JC-1 was prepared with the use of DMSO at a final concentration of $1 \mu \mathrm{mol} / \mathrm{L}$. This step was followed by incubation of cells in the dark for $30 \mathrm{~min}$. at $37^{\circ} \mathrm{C}$ and by further washing procedure, performed twice (5 min. $\times 2400$ rpm), using Ham's F-10 medium. The results were expressed as the percentage of cells exhibiting a high mitochondrial membrane potential.

\section{Detection of mitochondrial superoxide anion}

The amount of superoxide anion produced in the mitochondria of sperm cells was estimated with the use of Mi- 
Table 1. Effect of $17 \beta$-estradiol on spermatozoa vitality and phosphatydylserine membrane translocation $(\mathrm{N}=10)$

\begin{tabular}{|c|c|c|c|c|}
\hline & \multicolumn{4}{|c|}{ Type of spermatozoa } \\
\hline & $\begin{array}{c}\text { An-V-/PI- } \\
\text { Mean } \pm \text { SD (\%) }\end{array}$ & 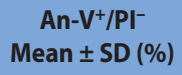 & $\begin{array}{c}\text { An-V-/PI+ } \\
\text { Mean } \pm \text { SD (\%) }\end{array}$ & 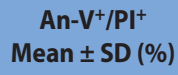 \\
\hline Control & $81.2 \pm 6.1$ & $0.4 \pm 0.3$ & $15.9 \pm 5.5$ & $2.4 \pm 1.4$ \\
\hline $\mathrm{E} 2\left[10^{-10} \mathrm{~mol} / \mathrm{L}\right]$ & $79.9 \pm 9.1$ & $1.2 \pm 1.2$ & $15 \pm 9.4$ & $3.8 \pm 1.9$ \\
\hline $\mathrm{E} 2\left[10^{-8} \mathrm{~mol} / \mathrm{L}\right]$ & $80.3 \pm 7.9$ & $0.8 \pm 0.8$ & $15.2 \pm 4.5$ & $3.6 \pm 1.3$ \\
\hline $\mathrm{E} 2\left[10^{-6} \mathrm{~mol} / \mathrm{L}\right]$ & $81.1 \pm 7.5$ & $0.7 \pm 0.6$ & $16.1 \pm 6.8$ & $2.2 \pm 1.1$ \\
\hline$P$ & $>0.05$ & $>0.05$ & $>0.05$ & $>0.05$ \\
\hline
\end{tabular}

E2 — 17ß-estradiol, An- $\mathrm{V}^{-} / \mathrm{PI}^{-}$- viable sperm without PST, An- $\mathrm{V}^{+} / \mathrm{PI}^{-}$- viable sperm with PST, $\mathrm{An}-\mathrm{V}^{-} / \mathrm{PI}^{+}$— dead sperm without PST, An- $\mathrm{V}^{+} / \mathrm{PI}^{+}$— dead sperm with PST, PST — phosphatydylserine membrane translocation, SD — standard deviation

toSOX Red fluorochrome (Molecular Probes Inc.), according to the method described by Koppers et al. [26]. The results were evaluated with the use of flow cytometer and confocal microscope. $5 \mathrm{mmol} / \mathrm{L}$ of MitoSOX Red were first diluted in DMSO in order to obtain stock solution, and then added to cell suspension $\left(20 \times 10^{6}\right.$ cells per $\mathrm{mL}$ ) to obtain the final concentration of $2 \mu \mathrm{mol} / \mathrm{L}$. This step was followed by a 15 minute-long cell incubation in the dark at $37^{\circ} \mathrm{C}$. After incubation, the samples were washed two times with medium F-10 (5 min. at $600 \times \mathrm{g}$ ) in order to perform microscopic and cytometric observations, conducted with the use of LSM 510 confocal microscope (Carl Zeiss GmbH, Germany). The fluorescence of MitoSOX Red was measured with the use of flow cytometer (Becton-Dickinson, USA). The results were expressed as the percentage of MitoSOX positive cells and as mean of fluorescence intensity of MitoSOX positive cells.

\section{Flow cytometry}

Flow cytometer FACSCalibur (Becton-Dickinson, USA) was used to analyze fluorescence signals of the labeled spermatozoa. 10,000 cells were examined per each experiment. The excitation wavelength used for obtaining fluorescence of An-V-FLUOS and PI was emitted by argon laser ( $488 \mathrm{~nm}$ ). The emission of the abovementioned dyes was then measured in either FL1 channel (515-545 nm, An-V-FLUOS, green fluorescence) or FL3 channel ( $650 \mathrm{~nm}, \mathrm{Pl}$, red fluorescence). The FL2 channel (561-603 nm) served for analyzing the fluorescence of MitoSOX Red. Fluorescence emitted by monomers and aggregates of JC- 1 was measured in the FL1 channel (515-545 nm) and FL2 channel (561-603 nm), respectively. All data were collected and analyzed using CellQuest Pro software (v.5.2.1) (Becton-Dickinson).

\section{Statistical analysis}

Statistica 10 software (StatSoft Inc., Tulsa, OK, USA) was used for statistical analysis. The following tests were applied: nonparametric U-Mann-Whitney and Kruskal-Wallis test with Dunn's post hoc tests. Data were considered statistically significant at $\mathrm{p}<0.05$ and presented as mean \pm SD.

\section{RESULTS}

\section{Sperm vitality and phosphatidylserine membrane translocation}

Based on the microscopic analysis and flow cytometric data, four sperm cells fractions were distinguished from the semen: (1) An-V/PI — viable sperm without PST, (2) An- $\mathrm{V}^{+} / \mathrm{PI}$ — viable sperm with PST, (3) An-V/PI+ — dead sperm without PST, and (4) An- $\mathrm{V}^{+} / \mathrm{PI}^{+}$— dead sperm with PST. Translocation of PS was most commonly observed within the cell membrane of the midpieces.

It has been shown that 2-hour sperm incubation with $17 \beta$-estradiol, genistein, or bisphenol-A did not reveal any statistically significant changes in both, the percentage of sperm fractions separated and the topography of the occurrence of the PS membrane translocation, regardless of the dose. Incubation of sperm cells with combination of all compounds tested at final concentrations of $10^{-10} \mathrm{~mol} / \mathrm{L}$ also did not significantly affect the viability or the PST (Table 1).

\section{Mitochondrial membrane potential}

Evaluation of $17 \beta$-estradiol, genistein, and bisphenol-A on human sperm mitochondrial membrane potential $\left(\Delta \Psi_{\mathrm{m}}\right)$ with flow cytometry technique was performed on the basis of staining with JC-1 fluorochrome. The percentage of sperm cells with high $\Delta \Psi_{\mathrm{m}}$ in the isolated fraction ranged between $60-90 \%$.

It has been shown that a two-hour incubation of sperm cells with E2 at a final concentration of $10^{-10} \mathrm{~mol} / \mathrm{L}$ does not cause any statistically significant changes of spermatozoa mitochondrial membrane $\Delta \Psi$. Incubation of sperm cells with $\mathrm{E} 2$ at a final concentration of $10^{-8} \mathrm{~mol} / \mathrm{L}$ caused a statistically significant increase in the percentage of sperm cells with high mitochondrial membrane $\Delta \Psi$ and a significant decrease in the percentage of spermatozoa with high mitochondrial membrane $\Delta \Psi$ at a final concentration of $10^{-6} \mathrm{~mol} / \mathrm{L}$ (Figure $1 \mathrm{~A}$ ).

Incubation of sperm cells with genistein, regardless of the dose used, did not cause statistically significant changes in sperm mitochondrial $\Delta \Psi$ (Figure 1B). 
A

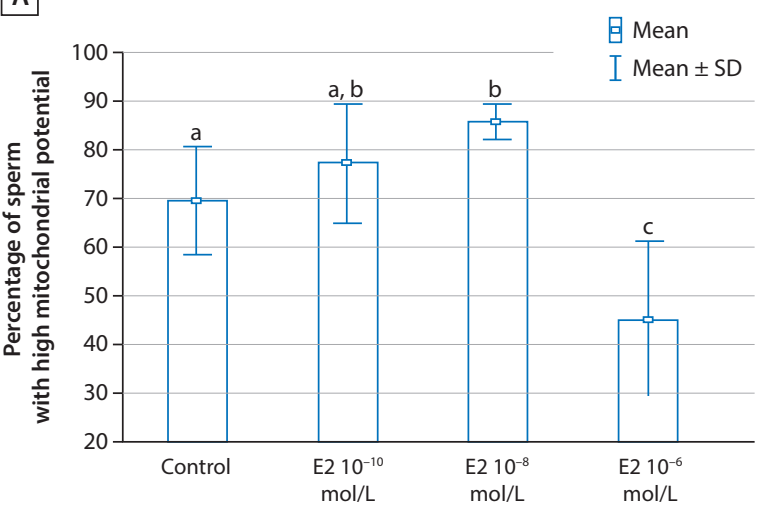

C

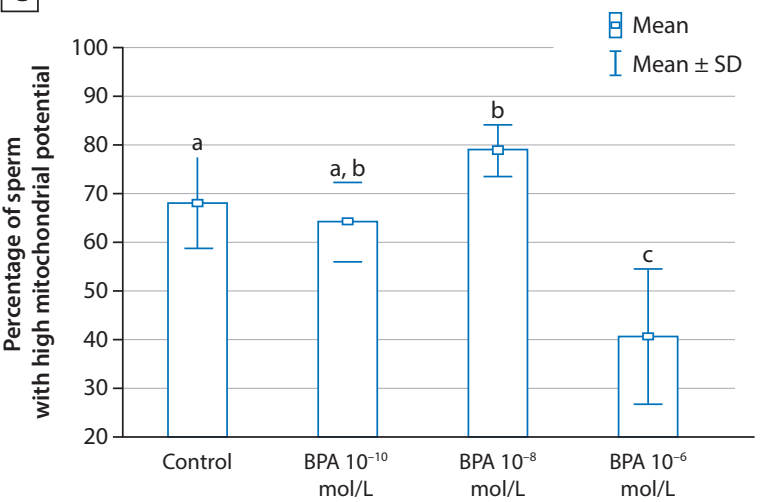

B

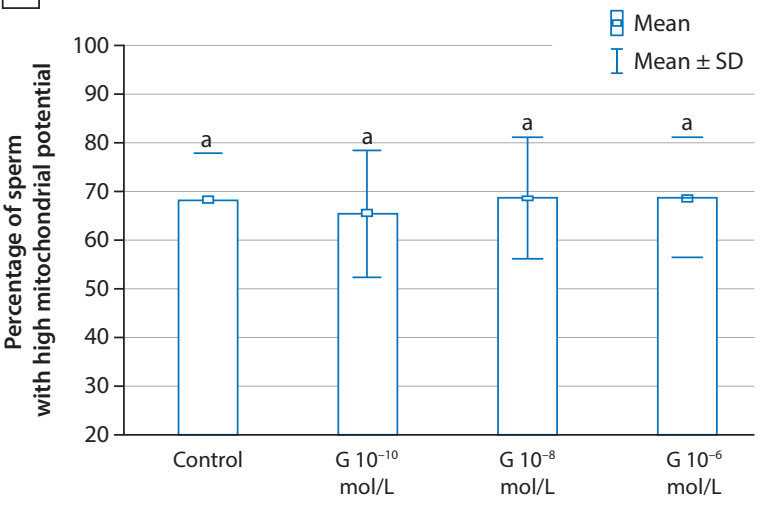

D

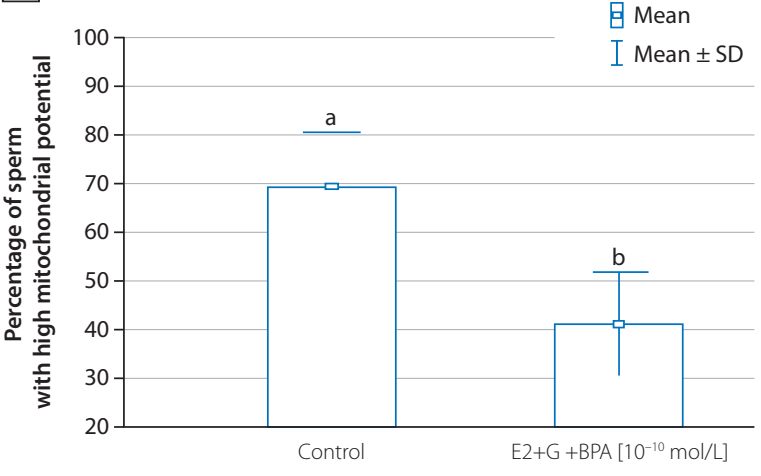

Figure 1. Analysis of the responses of spermatozoa mitochondrial membrane potential $\left(\Delta \Psi_{m}\right)$ to a 2-hour exposure to 17ß-estradiol (E2), genistein, and bisphenol-A (BPA). A. Significant dose-dependent changes in the percentage of spermatozoa with high mitochondrial membrane potential after a 2 hour-exposure to E2. B. No changes after genistein stimulation were observed. C. Significant dose-dependent changes in the percentage of spermatozoa with high mitochondrial membrane potential after a 2 hour-exposure to BPA. D. Significant changes in the percentage of spermatozoa with high $\Delta \Psi_{m}$ after a 2 hour-exposure to a mixture of E2, genistein, and BPA. Data obtained from 10 separate analyses are expressed as mean \pm standard deviation (SD). Different superscript letters above each bar vary significantly, $\mathrm{p}<0.05$

BPA, similarly to E2, at a final concentration of $10^{-10}$ did not cause a statistically significant change in sperm mitochondria $\Delta \Psi$, but at a final concentration of $10^{-8} \mathrm{~mol} / \mathrm{L}$ resulted in a statistically significant increase of the percentage of sperm cells with high mitochondrial membrane $\Delta \Psi(p=0.01)$. At a concentration of $10^{-6} \mathrm{~mol} / \mathrm{L}$, a statistically significant decrease in the percentage of sperm cells with high mitochondrial membrane $\Delta \Psi(p=0.01)$ was observed (Figure 1C).

Incubation of sperm cells with $17 \beta$-estradiol, genistein, and bisphenol-A at a final concentrations of $10^{-10} \mathrm{~mol} / \mathrm{L}$ caused a statistically significant decrease in the percentage of cells with high mitochondrial membrane $\Delta \Psi(p=0.0001)$ (Figure 1D).

\section{Detection of mitochondrial superoxide anion}

Evaluation of a two-hour incubation with $17 \beta$-estradiol, genistein, or bisphenol-A impact on sperm cells, did not show significant changes in the percentage of MitoSox positive sperm cells, regardless of the ligand type and dose ( $p>0.05$ ) (Table 2).
Sperm cell incubation with the combination of E2, genistein, and BPA at a concentration of $10^{-10} \mathrm{~mol} / \mathrm{L}$, caused a statistically significant increase in the percentage of MitoSOX positive cells $(38.0 \pm 8.3 \%$ vs. $27.7 \pm 12.1 ; p=0.03$ ) (Figure 2).

\section{DISCUSSION}

In our study, we analyzed the influence of $17 \beta$-estradiol, genistein, and bisphenol-A on sperm vitality, mitochondrial membrane potential, and production of mitochondrial superoxide anion. We did not prove any significant influence of the abovementioned compounds on the vitality of sperm cells. However, all of those ligands significantly modified mitochondrial function. The reaction depended on the concentration and type of the ligand.

There are only a few analyses which take under consideration the influence of estrogens and xenoestrogens on the vitality and apoptosis of mature spermatozoa. In our research, we analyzed the influence of $17 \beta$-estradiol, genistein, and bisphenol-A used at different concentra- 
Table 2. Percentage of MitoSOX positive sperm after 2 -hours incubation with $17 \beta$-estradiol, genistein or bisphenol-A ( $N=10)$

\begin{tabular}{|c|c|c|c|c|}
\hline & Mean & Minimum & Maximum & Standard deviation \\
\hline \multicolumn{5}{|c|}{$10^{-10} \mathrm{~mol} / \mathrm{L}$} \\
\hline Control & 27.7 & 6.7 & 42.0 & 12.1 \\
\hline $17 \beta$-estradiol & 25.4 & 13.1 & 41.7 & 12.5 \\
\hline Genistein & 29.0 & 7.5 & 57.2 & 18.9 \\
\hline Bisphenol A & 35.3 & 22.8 & 45.7 & 6.9 \\
\hline$P$ & $>0.05$ & $>0.05$ & $>0.05$ & $>0.05$ \\
\hline \multicolumn{5}{|c|}{$10^{-8} \mathrm{~mol} / \mathrm{L}$} \\
\hline Control & 27.7 & 6.7 & 42.0 & 12.1 \\
\hline $17 \beta$-estradiol & 31.7 & 13.7 & 59.4 & 16.1 \\
\hline Genistein & 33.1 & 13.5 & 68.3 & 19.8 \\
\hline Bisphenol A & 34.5 & 28.3 & 44.0 & 4.9 \\
\hline $\mathrm{P}$ & $>0.05$ & $>0.05$ & $>0.05$ & $>0.05$ \\
\hline \multicolumn{5}{|c|}{$10^{-6} \mathrm{~mol} / \mathrm{L}$} \\
\hline Control & 27.7 & 6.7 & 42.0 & 12.1 \\
\hline $17 \beta$-estradiol & 24.3 & 4.5 & 39.1 & 10.0 \\
\hline Genistein & 25.7 & 5.8 & 42.2 & 11.3 \\
\hline Bisphenol A & 37.5 & 24.5 & 54.7 & 9.5 \\
\hline $\mathrm{P}$ & $>0.05$ & $>0.05$ & $>0.05$ & $>0.05$ \\
\hline
\end{tabular}
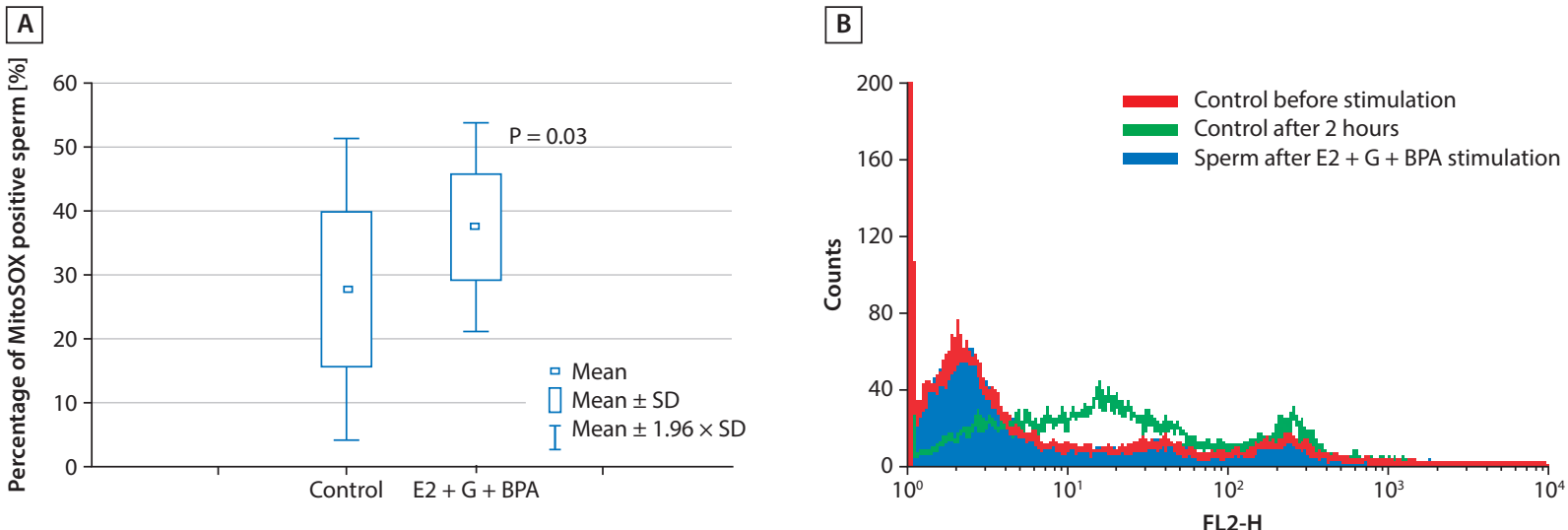

Figure 2. A. A significant increase in the percentage of MitoSOX-positive sperm after a 2 hour-exposure to a mixture of $17 \beta$-estradiol (E2), genistein, and bisphenol-A (BPA). B. Representative micrograph increase in intensity of MitoSOX fluorescence after E2, genistein, and BPA stimulation. Data obtained from 15 separate analyses are expressed as mean \pm standard deviation

tions on the vitality and PST of sperm cells, after a 2-hour incubation. We did not observe any statistically relevant changes in vitality and PST, regardless of the type of stimulus and its concentration. It can be assumed that the analyzed substrates do not affect those processes in sperm cells. In a study by Hinsch et al. [27], xenoestrogens did not significantly affect the vitality of spermatozoa. They analyzed the influence of genistein on bull spermatozoa obtained with the use of the swim-up isolation. No significant changes of either vitality or motility were demonstrated. However, incubation of spermatozoa with genistein at small concen- trations caused a significant, dose-dependent decrease in the percentage of spermatozoa able to penetrate the zona pellucida. These authors suggest that stimulation of sperm cells with genistein can influence their ability to fertilize the egg, without changing their viability [27].

Our research did not prove a statistically significant influence of genistein, either on sperm vitality or mitochondrial function. However, other reports indicate that genistein seems to affect spermatozoa in a dose-depend manner, and probably the reaction differs between species. For example, in a study by Martinez-Soto et al. [28], genistein was 
hypothesized to possess antioxidant properties and to be able to protect spermatozoa DNA, endangered by damage resulting from oxidative stress. Next, to check the changes in cell quality, these authors analyzed the influence of supplementation of medium normally used for cryopreservation of spermatozoa, modified by adding genistein at a final concentration of $10 \mu \mathrm{mol} / \mathrm{L}$. After thawing, they observed the following changes: (1) a slight increase of motility parameters, (2) a decrease of disturbances in membrane lipid structure, and (3) a decreased level of DNA damage. Those results confirm the antioxidative influence of genistein and its ability of protect DNA [29]. These authors conclude that their observations can be useful in preparing semen for IVF procedures [28].

Although in our study genistein did not significantly alter the level of mitochondrial superoxide anion, it cannot be excluded that those changes actually occurred. It should be pointed out that in our study the concentration of the analyzed anion and its fluorescence levels could be too low to be observed with use of the applied techniques due to two reasons: 1) the amount of mitochondria present in spermatozoa is low and 2) we analyzed sperm cells isolated with use of the swim-up technique and obtained from normozoospermic men.

Various authors suggest that the increased exposition to BPA correlates with lower sperm quality measures [30]. BPA levels were also examined in follicular fluid [31] and human semen [32], and were estimated at $1.0-2.0 \mathrm{ng} / \mathrm{mL}$ and $5.1 \mathrm{ng} / \mathrm{mL}$, respectively. Surprisingly, BPA was present also in the amniotic fluid at an approximately 5 -fold higher concentration $(8.3 \pm 8.7 \mathrm{ng} / \mathrm{mL})$. These results suggest a probable influence of BPA not only on fetuses after early exposure to this compound during the prenatal period, but also on the biology of mature sperm cells at different stages of development.

Our study indicates that both, E2 and BPA at a concentration of $10^{-8} \mathrm{~mol} / \mathrm{L}$ caused a distinct increase in the percentage of spermatozoa with high $\Delta \Psi_{\mathrm{m}}$. However, a significant decrease in spermatozoa with high $\Delta \Psi_{\mathrm{m}}$ was observed in case of both compounds at a concentration of $10^{-6} \mathrm{~mol} / \mathrm{L}$. Incubation of sperm cells with E2, genistein, and BPA at a final concentration of $10^{-10} \mathrm{~mol} / \mathrm{L}$ resulted in a greater decrease in $\triangle \Psi_{\mathrm{m}}$ than stimulation with the use of only one factor, E2 or BPA, at a concentration of $10^{-6} \mathrm{~mol} / \mathrm{L}$.

E2 and xenoestrogens have been suggested to stimulate the increase in intracellular $\mathrm{Ca}^{2+}$ level [33-35]. It is proposed that the increase in calcium ion concentration stimulated by estrogens may activate mitochondrial protein phosphatase, which in turn dephosphorylates cytochrome c oxidase. As a consequence, membrane mitochondrial potential, as well as the amount of ROS, may increase [36]. It can be assumed that changes in $\Delta \Psi_{\mathrm{m}}$ stimulated by $\mathrm{E} 2$ and xen- oestrogens can be caused by influx of calcium ions into the mitochondria. In our study, low concentrations of ligands caused an increase in $\Delta \Psi \mathrm{m}$. However, high doses of $E_{2}$ and BPA or stimulation of sperm cells with the mixture of the compounds caused the decrease in $\Delta \Psi_{\mathrm{m}}$. The excessive increase in mitochondrial calcium ions concentration, which may be observed as an effect of synergistic action of used compounds, can be the cause of decrease in mitochondrial membrane potential observed in this study.

A decrease in $\Delta \Psi_{\mathrm{m}}$ might also be the consequence of oxidative stress. There are data indicating that some metabolites of xenoestrogens and estradiol may lead to oxidative stress in human sperm cells. In case of genistein, only high doses caused ROS production. Some estrogen and benzene derivatives (diethylstilbestrol and catechol, respectively) caused intense ROS production. However, no significant changes were observed in case of compounds such as $17 \beta$-estradiol, nonyphenol or bisphenol-A [36].

In conclusion, estrogens and xenoestrogens analyzed in this study affect the mitochondrial function. The observed effect depends on substrate type and its concentration. It seems that estrogens and genistein, occurring in physiological doses, take part in the regulation of mitochondrial function and may play the role of antioxidant factors. Our findings, in which spermatozoa were stimulated with the mixture of the three substrates or with either $17 \beta$-estradiol or bisphenol-A at high concentrations, suggest that excessive exposition of spermatozoa to such types of substrates may negatively affect the mitochondria of human sperm cells and, in turn, reduce male fertility potential.

\section{ACKNOWLEDGMENTS}

The National Research Centre grant N N 401077037 funds were received in support of this work.

\section{REFERENCES}

1. Bonde JP. Male reproductive organs are at risk from environmental hazards. Asian J Androl. 2010, 12, 152-156.

2. Machtinger R, Orvieto R. Bisphenol A, oocyte maturation, implantation, and IVF outcome: review of animal and human data. Reprod BioMed Online. 2014, 29, 404-410.

3. Phillips KP, Tanphaichitr N. Human exposure to endocrine disrupters and semen quality. J Toxicol Environ Health B Crit Rev. 2008, 11, 188-220.

4. Chambliss KL, Yuhanna IS, Mineo C, [et al.]. Estrogen receptor alpha and endothelial nitric oxide synthase are organized into a functional signaling module in caveolae. Circ Res. 2000, 87, 44-52.

5. Viñas R, Jeng YJ, Watson CS. Non-genomic effects of xenoestrogene mixtures. Int J Environ Res Public Health. 2012, 9, 2694-2714.

6. Rajender S, Rahul P, Mahdi AA. Mitochondria, spermatogenesis and male infertility. Mitochondrion. 2010, 10, 419-428.

7. Tavares RS, Martins FC, Oliveira PJ, [et al.]. Parabens in male infertility - is there a mitochondrial connection? Reprod Toxicol. 2009, 27, 1-7.

8. Felty Q, Roy D. Estrogen, mitochondria and growth of cancer and non-cancer cells. J Carcinog. 2005, 4, 1-18.

9. Psarra AM, Sekeris CE. Nuclear receptors and other nuclear transcription factors in mitochondria: regulatory molecules in a new environment. Biochim Biophys Acta. 2008, 1783, 1-11.

10. Chen JQ, Eshete M, Alworth WL, [e al.]. Binding of MCF-7 cell mitochondrial proteins and recombinant human estrogen receptors alpha and 
beta to human mitochondrial DNA estrogen response elements. J Cell Biochem. 2004, 93, 358-373.

11. Gavrilova-Jordan LP, Price TM. Actions of steroids in mitochondria. Semin Reprod Med. 2007, 25, 154-164.

12. Rachamim N, Latter H, Malinin N, [et al.]. Dexamethasone enhances expression of mitochondrial oxidative phosphorylation genes in rat distal colon. Am J Physiol. 1995, 269, C1305-C1310.

13. Tatoyan A, Giulivi C. Purification and characterization of a nitricoxide synthase from rat liver mitochondria. J Biol Chem. 1998, 273, 11044-11048.

14. Walczak-Jedrzejowska R. Oxidative stress and male infertility. Part I: Factors causing oxidative stress semen. Post Androl Online. 2015, 2 (1), 5-15.

15. Postawski K, Przadka-Rabaniuk D, Piersiak T. 8-oxo-7,8-dihydroguanine level — the DNA oxidative stress marker — recognized by fluorescence image analysis in sporadic uterine adenocarcinomas in women. Ginekol Pol. 2013, 84 (1), 44-50.

16. Aquila $S$, Sisci $D$, Gentile $M$, [et al.]. Estrogen receptor (ER) alpha and ER beta are both expressed in human ejaculated spermatozoa: evidence of their direct interaction with phosphatidylinositol-3-OH kinase/Akt pathway. J Clin Endocrinol Metab. 2004, 89, 1443-1451.

17. Rago V, Siciliano L, Aquila S, Carpino A. Detection of estrogen receptors ER-alpha and ER-beta in human ejaculated immature spermatozoa with excess residual cytoplasm. Reprod Biol Endocrinol. 2006, 4, 36.

18. Solakidi S, Psarra AM, Nikolaropoulos S, [et al.]. Estrogen receptors alpha and beta (ERalpha and ERbeta) and androgen receptor (AR) in human sperm: localization of ERbeta and AR in mitochondria of the midpiece. Hum Reprod. 2005, 20, 3481-3487.

19. Guido C, Perrotta I, Panza S, [et al.]. Human sperm physiology: estrogen receptor alpha $(E R a)$ and estrogen receptor beta $(E R \beta)$ influence sperm metabolism and may be involved in the pathophysiology of varicocele-associated male infertility. J Cell Physiol. 2011, 226, 3403-3412.

20. Vandenberg LN, Hauser R, Marcus $M$, [et al.]. Human exposure to bisphenol A (BPA). Reprod Toxicol. 2007, 24, 139-177.

21. Cederroth CR, Zimmermann C, Nef S. Soy, phytoestrogens and their impact on reproductive health. Mol Cell Endocrinol. 2012, 355 (2), 192-200.

22. Whitten PL, Patisaul HB. Cross-species and interassay comparisons of phytoestrogen action. Environ Health Perspect. 2001, 1, 5-20.

23. Kuiper GG, Lemmen JG, Carlsson B, [et al.]. Interaction of Estrogenic Chemicals and Phytoestrogens with Estrogen Receptor beta. Endocrinology. 1998, 139, 4252-4263.
24. WHO laboratory manual for examination and processing of human semen. Fifth Edition. WHO Press 2010.

25. Jameel T. Sperm swim-up: a simple and effective technique of semen processing for intrauterine insemination. J Pak Med Assoc. 2008, 58, 71-74.

26. Koppers AJ, De luliis GN, Finnie JM, [et al.]. Significance of mitochondrial reactive oxygen species in the generation of oxidative stress in spermatozoa. J Clin Endocrinol Metab. 2008, 93, 3199-3207.

27. Hinsch KD, Aires V, Hägele W, [et al.]. In vitro tests for essential sperm functions using the phyto-oestrogen genistein as a test substance. Andrologia. 2000, 32, 225-231.

28. Martinez-Soto JC, de DiosHourcade J, Gutiérrez-Adán A, [et al.]. Effect of genistein supplementation of thawing medium on characteristics of frozen human spermatozoa. Asian J Androl. 2010, 12, 431-441.

29. Sierens J, Hartley JA, Campbell MJ, [et al.]. Effect of phytoestrogen and antioxidant supplementation on oxidative DNA damage assessed using the comet assay. Mutat Res. 2001, 485, 169-176.

30. Li DK, Zhou Z, Miao M, [et al.]. Urine bisphenol-A (BPA) level in relation to semen quality. Fertil Steril. 2011, 95, 625-630.

31. Ikezuki Y, Tsutsumi O, Takai Y, [et al.]. Determination of bisphenol A concentrations in human biological fluids reveals significant early prenatal exposure. Hum Reprod. 2002, 17, 2839-2841.

32. Inoue $\mathrm{K}$, Wada $\mathrm{M}$, Higuchi $\mathrm{T}$, [et al.]. Application of liquid chromatography-mass spectrometry to the quantification of bisphenol $A$ in human semen. J Chromatogr B Analyt Technol Biomed Life Sci. 2002, 773, 97-102.

33. Campagna $C$, Guillemette $C$, Ayotte $P$, [et al.]. Effects of an environmentally relevant organochlorine mixture and a metabolized extract of this mixture on porcine sperm parameters in vitro. J Androl. 2009, 30, 317-324.

34. Tavares RS, Mansell S, Barratt CLR, [et al.]. p,p'-DDE activates CatSperm and compromises human sperm function at environmentally relevant concentrations. Hum Reprod. 2013, 28, 3167-3177.

35. Kotula-Balak M, Tworzydlo W, Pochec E, [et al.]. Octylphenol induces changes in glycosylation pattern, calcium level and ultrastructure of bank vole spermatozoa in vitro. Toxicol In Vitro. 2015, 29 (3), 529-537.

36. Bennetts LE, De luliis GN, Nixon B, [et al.]. Impact of estrogenic compounds on DNA integrity in human spermatozoa: evidence for cross-linking and redox cycling activities. Mutat Res. 2008, 641, 1-11. 